\title{
Estimation of the probability of karst cavities collapse under technogenic cyclic loads below the fatigue limit
}

\author{
$O R$ Kuzichkin $^{1, *}, N V$ Dorofeev $^{1}, A V$ Grecheneva $^{1}, R V$ Romanov $^{2}$, and $D I$ Surzhik $^{2}$ \\ ${ }^{1}$ Belgorod State University, 85 Pobedy St, Belgorod, 308015, Russia \\ ${ }^{2}$ Vladimir State University, 87 Gor'kogo St, Vladimir, 600000, Russia
}

\begin{abstract}
The paper deals with the phenomenon of fatigue formation in soils over karst cavities under cyclic loading near the fatigue limit. A method is proposed for determining the stress state of the integumentary earth arising during the considered technogenic cyclic loads. Currently, the assessment of the possibility of karst cavities collapse is performed on the basis of static models, without taking into account the dynamic technogenic load. Especially in the case of slow cyclic loads of low intensity, which do not directly lead to collapse of the soil above the karst cavities. However, these actions change the bearing capacity of soils that are not taken into account at the exploration stage and are not laid in the project documentation for the construction of facilities in karst-dangerous areas. A technique for estimating pre-crisis states based on joint processing of active geoelectric and seismoacoustic geodynamic control signals is proposed.
\end{abstract}

\section{Introduction}

The tests of soils under dynamic loads are the necessary condition for assessing changes in the properties of soils during their operation when cyclic loads of natural and technogenic nature are applied to them [1]. A serious problem is the prediction of the stability of the soil bases of structures in the presence of a covered karst. In this case, the collapse of the karst cavities is characterized by the spontaneity of occurrence and significantly complicates the safety of the objects $[2,3]$. For prediction of destruction and protection of operation of buildings at the design stage of construction, calculations of the bearing properties of soil in the karst territory using static models are carried out [4]. However, the absence of a unified theory of taking into account the parameters of soil rock fatigue does not allow to fully adapting the calculation methods used to solve the problems of determining the fatigue limit of inhomogeneous media (soil). At present, they are still widely used in various methods for determining the fatigue limit of soils, the theoretical level of which varies from $48 \%$ to $70 \%$ of the static strength [5].

Under the fatigue limit is meant a decrease in strength properties and a significant increase in the deformability of the soil, leading to its destruction. From the point of view

\footnotetext{
* Corresponding author: 1155464@bsu.edu.ru
} 
of prediction and prevention of emergency situations of soil collapses, it is interesting to analyze the strength properties of the soil stratum under conditions of man-made cyclic loads below the fatigue limit. This type of load is dynamic and most common and is characterized by a constant cyclic impact associated with the operation of buildings, the movement of traffic stream, the operation of underground communications [6]. Since it is impossible to predict changes in the level of man-caused load in the process of building up areas, the calculated static models used in the initial stages of construction often lose their relevance and do not allow estimating the changes in the strength properties of the soil foundation in the mode of changing (increasing) technogenic impacts. It is especially important to take into account the slow cyclic loads of low intensity, which do not directly lead to collapse of the soil over the karst cavities, but have a significant impact on the change in its strength properties, which were determined at the stage of primary engineering surveys and specified in the design documentation. Therefore, the actual task is to estimate the probability of collapse of karst cavities under technogenic cyclic loads below the fatigue limit, the solution of which is the subject of this article.

\section{Processes in soils over karst cavities under cyclic loading near the fatigue limit}

Structural features of the karsting environment are the presence of microcracks (inclusion of weak minerals, the presence of pores of different configurations). Consequently, under the influence of cyclic loading, the stress field is redistributed into the primary field propagating in the medium, and the secondary, concentrating in the microinhomogeneity localization zones [7]. The secondary stress field is characterized by variability in each of the cycles, since from the point of view of load distribution, microcracks are the most vulnerable areas of stress concentration. Each cyclic action below the limit of soil fatigue leads to the accumulation of plastic deformations and the slow growth of microcracks. This process is accompanied by the convergence of the vertices of growing microcracks to a certain threshold (some critical distance), in excess of which the microcracks merge to form a single microcavity. Thus, cyclic loading creates favorable conditions for the concentration of stresses at the weakest points of the karsting medium, which leads to its destruction at substantially lower cyclic loads than under static conditions adopted in construction calculation models.
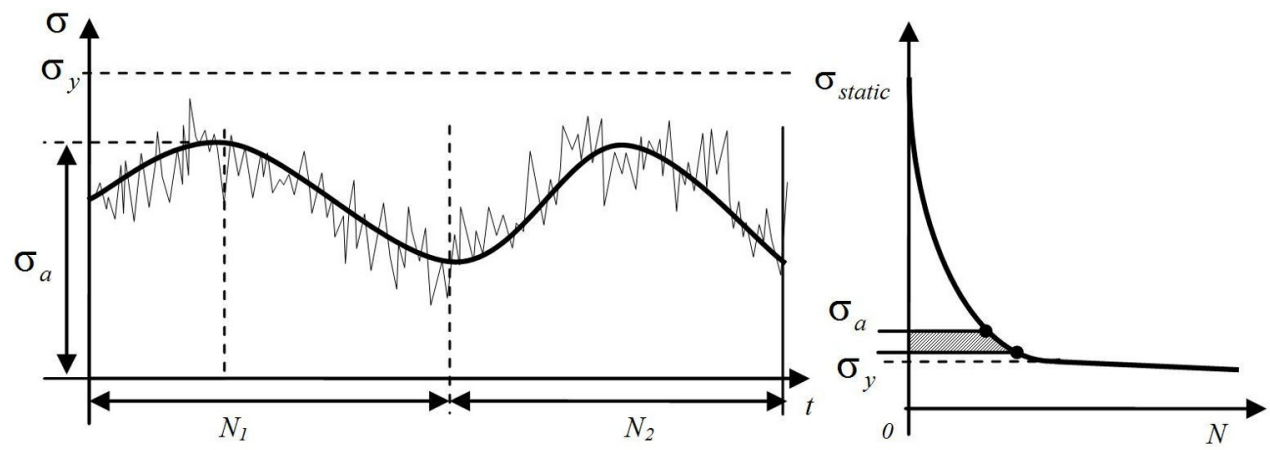

Fig. 1. The model of the formation of cyclic loads in a soil massif.

Under the conditions of the nonequilibrium state caused by the impact of cyclic loads leading to the formation of microcracks in the karst massif, the cover layer of the deposits acts as a typical dissipative system capable of evolving and creating ordered structures (in this case, deformation ones) caused by the process of self-organization of the geological 
environment [8]. The change in the state and strength properties of soils in the roof part of the cavity, associated with the unloading of stresses at the points of weakening of the karst massif, leads to deformation of the integumentary thickness of the karst cavity and its expansion, and can initiate the collapse of the arch of the cavity up to its exit to the surface (in the form of a dip). The model for the formation of cyclic loads in an array is shown in Figure 1.

The model of cyclic technogenic impact on the geological environment can be described as a pulsating load with a sinusoidal waveform. The periods of loading and unloading are commensurable with periods of increased activity of anthropogenic load (for example, diurnal changes in traffic density of transport stream). It should be noted that as the number of load cycles $N$ increases with the maximum value of the cycle stress $\sigma_{a}$, the probability of occurrence of deformation of the soil increases, as the values $\sigma_{a}$ approach the values of the fatigue limit $\sigma_{y}$.

The kinetic (thermofluctuation) theory of the destruction of solids [9] is based on the fact that in solid bodies (which in the first approximation is the soil foundation) under the influence of cyclic loads, the accumulation of damages continuously proceeds, which leads to complete destruction of the solids. The time of the non-critical deformable state of the soil base under the action of cyclic loading is determined by the following formula:

$$
t=t_{0} \exp \cdot \frac{\left(U_{0}-\sigma V\right)}{k T},
$$

where $t_{0}$ is the constant that coincides in order with the period of cyclic oscillations and is independent of the chemical composition and structure of the solid, $U_{0}$ is the activation energy of the process of destruction; $V$ is the activation volume, depending on the structure of the solids, $k T$ is the measure of the energy of thermal fluctuations, $k$ is the Boltzmann constant, $T$ is the absolute temperature of the soil.

Since in karst rocks contacts between particles can have different strengths, and consequently, different activation energies, one can assume the presence of a whole series of energy steps from $E_{a k t-1}$ to $E_{a k t-n}$ levels. For a macroscopically discernible reduction in strength at loads below the fatigue limit, the presence in the rock of a certain critical number of inhomogeneities with the activation energy below 1 level is necessary. Then, the probability of activation of karst processes is in the zone with the activation energy below 1 level (Figure 2).

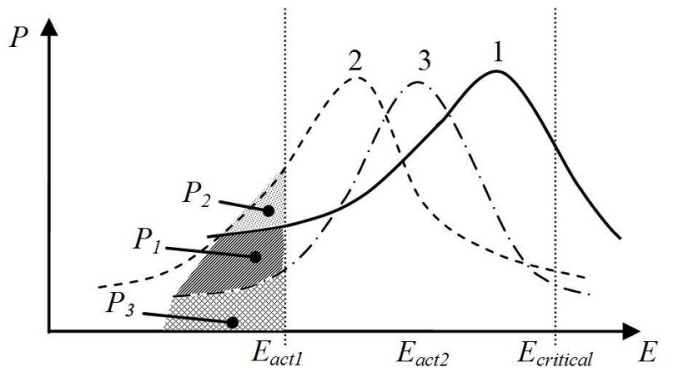

Fig. 2. The probability of reducing the strength of the soil in zones less than the activation energy of the first level.

The probability $P$ of the existence of such weak inhomogeneities in nature is expressed by the shaded areas $P_{1}, P_{2}, P_{3}$ under the distribution curves. Behind the upper limit of the confidence interval are the critical values of the activation energy, which almost 
inevitably lead to the destruction of the rock (the condition of static destruction). The probability of failure because of the mechanical fatigue depends on the likelihood of nearby several cracks in which stresses can reach a value equal to the limit of brittle strength. Such a probability increases with the growth of the size of the karstic environment. The greater the volume of karstic rock, the lower the fatigue limit and the greater the probability of weaknesses. Obviously, the achievement of activation energy close to the mode of any of these distributions means the possibility of destruction of the karstic rock. Therefore, the activation energy of the first level, reached at voltages that do not lead to destruction (less fatigue limit), can be only beyond the limits of the confidence interval of the mean activation energy in the lower quartile.

\section{Assessment of pre-crisis states on the basis of joint processing of signals of the active geoelectric and seismoacoustic geodynamic control}

The model of the processes of the stress-strain state of the soil, depending on the load, can be broken down into several phases [10]. In this case, three main phases of the stress-strain process at the foundation of the building are distinguished. The phase of elastic deformation (I) (compaction phase) is limited by the value of the structural strength $P_{\text {str }}$, assuming that structural loads do not occur under these loads in the soil. With increasing load on the soil foundation, structural geodynamic changes in the medium, characterized by the phases of the shifts (II) and the bulge (III), begin.

The task of detection the initial stage of irreversible geodynamic changes at the foundation of the building above the karst cavity consists in the separation of the shear phase, which is characterized by significant shear strains, due to the excess of the CoulombMohr strength limit. The procedure for detection the preliminary fracture phase is based on the recording of a change in the electrical resistance of the medium under the influence of elastic vibrations. It is established that the change in the electrical resistance of media under the influence of elastic action (seismoelectric effect of the first kind) is due to the presence of microcracks in them, which corresponds to the initial phase of destruction of soil foundation of buildings and of the soil above the karst cavities.

Based on the formulas described in [11], under the deformation effect of a longitudinal wave, the electrical parameters of the medium will take the following form:

$$
R_{x}{ }^{\prime}=\rho \frac{l_{x}\left(1+u_{x}\right)}{S_{y z}} ; C_{x}{ }^{\prime}=\varepsilon \frac{S_{y z}}{l_{x}\left(1+u_{x}\right)},
$$

where $R_{x}{ }^{\prime}, C_{x}{ }^{\prime}$ are the electrical parameters of the geological medium, $u_{x}$ is the element of the strain tensor in $x, S_{y z}$ is the cross-sectional area of the controlled sample of the medium, $l_{x}$ is the length of the medium sample, $\rho$ is the resistivity of the geological medium, and $\varepsilon$ is the dielectric constant of the geological medium. When checking in the area of foundation of the building above the karst cavities, the geoelectric section can be represented as a model for dividing the two (or anymore) media - the deformed part and the non-deformable part with parameters $\rho_{1}, \varepsilon_{1}$ and $\rho_{2}, \varepsilon_{2}$ located at a distance $a=k \Delta x$ from the origin, where $l<k<n$. At the same time, the transfer coefficient of the geoelectric section in complex form: 


$$
H(j \omega)=\frac{l_{x}}{S_{y z}} \cdot\left\{\begin{array}{l}
\frac{\rho_{1}}{1+j \omega \rho_{1} \varepsilon_{1}} \sum_{i=1}^{k=1}\left(1+u_{x}{ }^{i}\right)+ \\
+\frac{\rho_{2}}{1+j \omega \rho_{2} \varepsilon_{2}} \sum_{i=k}^{n}\left(1+u_{x}{ }^{i}\right)
\end{array}\right\},
$$

where $\omega$ is the frequency of the probing geoelectric signal.

As shown from the above relation (3), only the fundamental harmonics of the probing effects will be present in the spectrum of the geoelectric signal in the absence of plastic deformations. However, in the transition from the elastic stage to the plastic deformation stage, the combinational frequencies of the seismoelectric effect will manifest in the spectrum of the geoelectric signal.

\section{Results and conclusions}

To study the impact of cyclic fatigue in soils and to establish a connection between the electrical and elastic parameters of rocks, experimental studies were carried out using a specially designed strain gauge. Soil samples having an identical homogeneous macroscopic and isotropic structure were investigated. The studies were carried out in two stages: in the first stage, the sample was loaded with a constant load $G_{\text {const }}$, characterizing the external load from the engineering structures, during the time $t$ at which the sample reached the fatigue limit Pf and was destroyed (Figure $3 \mathrm{a}$ ). In the second stage, the identical sample was subjected to a constant $G_{\text {const }}$ load and an additional cyclic $G_{c y c l i c}$ load, below the fatigue limit Pf '(Figure 3b). The strain of the samples was measured using a special tensometric device. The registration of the ohmic resistance and the relative deformation of the experimental sample are carried out using a block of amplifiers and a source of stable voltage.

Experimental studies have shown that the relative deformation of the soil sample and the change in the measured resistance $\Delta R$ vary linearly. Moreover, in the case of the impact of an additional cyclic load, a cyclic trend of the change in soil resistance is observed. The change in resistance $\Delta R$ decreases linearly with increasing pressure on the soil sample in both cases to a certain limit. The change in the course of $\Delta R$ is related to the formation of cracks in an isotropic medium, which corresponds to the appearance of nonlinear processes in the sample before its destruction. In the absence of cyclic loading, when the fatigue limit reaches $P_{f}=20 \mathrm{~kg} / \mathrm{cm}^{3}$, the resistance changes deviate from the linear stroke. In the case of an additional effect of the cyclic load $G_{\text {cyclic }}$, the attainment of the fatigue limit occurs at lower load limit values $\quad P_{f}^{\prime}=17 \mathrm{~kg} / \mathrm{cm}^{3}$ and depends on the number of cycles $N$.

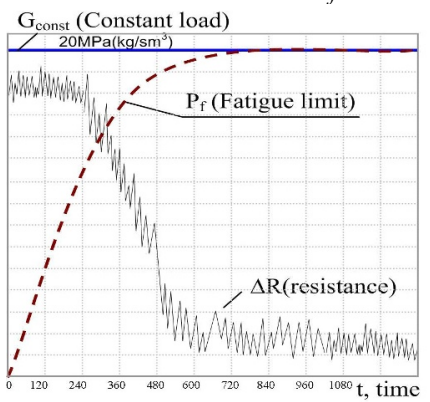

a)

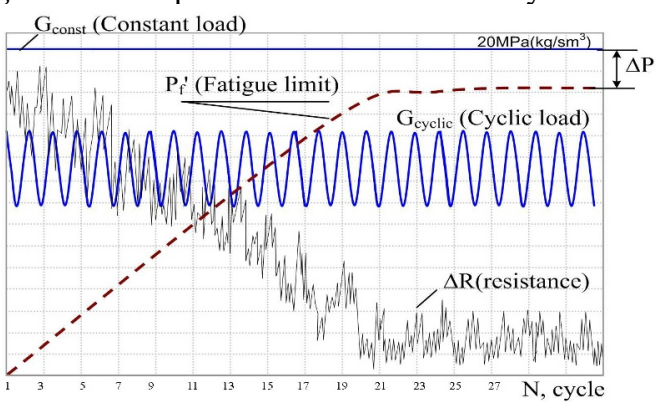

b)

Fig. 3. The results of experimental studies. 
It is established that the probability of failure of the karst cavity is determined by the value of $\Delta P$, which can be modeled for each soil type and taking into account the external cyclic load below the fatigue limit. In this case, it is established that in the case of cyclic loading, the probability of errors of the first and second kind is determined from the formula, and will be less than $12.4 \%$ :

$$
P_{(1,0)}=P_{(0,1)}=\sum_{N}^{i=1} \frac{P_{f}-P_{f}^{\prime}}{P_{f}^{\prime}}=\sum_{N}^{i=1} \frac{\Delta P}{P_{f}^{\prime}} \leq 12,4 \%
$$

Consequently, structural geodynamic changes in the karst cover can be detected by geoelectrical methods earlier than irreversible geodynamic destruction of the object. It is proved that cyclical technogenic loads below the limits of soil fatigue lead to a high probability of failure of karst cavities. The probability of failure because of the mechanical fatigue depends on the likelihood of nearby several cracks in which stresses can reach a value equal to the limit of brittle strength. Such a probability increases with the growth of the size of the karstic environment. The greater the volume of karstic rock, the lower the fatigue limit and the greater the probability of weaknesses.

\section{Acknowledgments}

The work was carried out as part of the research on the project, support by the Ministry of Education and Science of the Russian Federation No. 5.3606.2017/PCH.

\section{References}

1. Joseph Paul G 2017 Dynamical Systems-Based Soil Mechanics (CRC Press/Balkema) p 138

2. Ford D C and Williams P 2007 Karst Hydrogeology and Geomorphology, (John Wiley and Sons Ltd) pp 43-52

3. An Beynen P 2011 Karst management (Springer) pp 12-18

4. Ford Derek 2007 Jovan Cvijić and the founding of karst geomorphology (Environmental Geology) 51 pp 675-684

5. McCarthy David F 2006 Essentials of soil mechanics and foundations: basic geotechnics (Upper Saddle River, New Jersey: Prentice Hall) pp 33-40

6. Jefferies Mike and Been Ken 2006 Soil Liquefaction: A Critical State Approach (Taylor \& Francis) pp 65-70

7. Voznesensky E A, Remenyak K M and Semidetko I V 1992 Behavior of rocks under cyclic loads below the fatigue limit (Engineering Geology) 2 pp 38-49

8. Anikeev A V, Kalinin E V and Tarakanov S I 1991 Determination of the stress state of the soil strata over the karst cavity (Engineering Geology) 5 pp 64-70

9. Regel V R, Slutsker A I and Tomashevsky E E 1974 The kinetic nature of the strength of solids (Moscow, Science) p 560

10. Joseph Paul G 2014 Generalized soil deformation model based on dynamical systems theory (Geotechnical Research) 1 pp 32-42

11. Bykov A, Kuzichkin O and Dorofeev N 2015 The Resistive-Acoustic Method of Monitoring Industrial Sludge Plume (Applied Mechanics and Materials) 770 pp 679685 